\section{Sudden Death of a Woman \\ at Postpartum Period - Myocarditis Related with Peripartum Cardiomyopathy: Autopsy Diagnosis, Case Report}

\section{Burcu Kasap ${ }^{1 *}$, Emre Canverenler ${ }^{2}$, Kenan Karbeyaz ${ }^{3}$, Yasemin Balcı $I^{4,5}$ and Melike Erbaş ${ }^{5}$}

${ }^{1}$ Department of Obstetrics and Gynecology, School of Medicine, MuğlaSıtkıKoçman University, Menteşe, Muğla, Turkey

${ }^{2}$ Department of Obstetrics and Gynecology, Sinop State Hospital, Sinop, Turkey

${ }^{3}$ Justice Ministry, Institute of Forensic Medicine, Eskişehir, Turkey

${ }^{4}$ Department of Forensic Medicine, School of Medicine, MuğlaSıtkıKoçman University, Menteşe, Muğla, Turkey

${ }^{5} J u s t i c e$ Ministry, Institute of Forensic Medicine, Muğla, Turkey

\begin{abstract}
A 29-year-old woman underwent cesarean section for twin pregnancy due to previous cesarean section operation. The patient died suddenly at postpartum eight day and the medico-legal autopsy results revealed 'focal myocarditis'. Since the patient had no symptoms or signs of a cardiac disorder during the hospitalization, focal myocarditis was regarded as a complication rather than the cause. Herein, we report the sudden death of a woman at postpartum period and discuss the differential diagnosis by reviewing the related literature.
\end{abstract}

Keywords: Autopsy; Focal myocarditis; Maternal death

*Corresponding author: Burcu Kasap, Department of Obstetrics and Gynecology, School of Medicine, MuğlaSıtkıKoçman University, Menteşe, Muğla 48000, Turkey, Tel: +90 25221148005156; E-mail: burcuharmandar@gmail.com; burcuharmandar@mu.edu.tr

Citation: Kasap B, Canverenler E, Karbeyaz K, Balcı Y, Erbaş M (2015) Sudden Death of a Woman at Postpartum Period - Myocarditis Related with Peripartum Cardiomyopathy: Autopsy Diagnosis, Case Report. J Clin Stud Med Case Rep 2: 009.

Received: December 30, 2014; Accepted: February 25, 2015; Published: March 11, 2015

\section{Introduction}

Maternal mortality is the death of a woman during pregnancy and within 42 days after delivery. The most frequent direct or indirect diagnoses associated with maternal mortality are; pregnancy-induced hypertension, pulmonary embolus, amniotic fluid embolus, hemorrhage and cardiac disease [1]. The distribution of cardiac disease related maternal deaths differ in reasons from country to country. Myocardial infarction, cardiomyopathy and Congenital Heart Diseases (CHD) are the leading causes in western countries whereas rheumatic heart diseases are accused mostly in developing countries [2]. The incidence of cardiovascular diseases in pregnancy is about 2-4\% [3]. Myocarditis is the inflammation of the myocardium due to variable etiologies and $2.5 \%$ of the maternal deaths were caused by myocarditis or myocardial fibrosis [4]. Herein, we present a rare case of sudden maternal death which was complicated with focal myocarditis' according to autopsy report.

\section{Case Presentation}

A 29-year-old (gravid 3; para 2) woman who had 35 weeks-old twin pregnancy was referred to our hospital due to spontaneous progression of the labor. In her admission, ultrasonographic examination revealed 35 weeks of viable twin pregnancy without any notable pathology. In cardiotocography, spontaneous and regular contractions were present with reassuring fetal heart rates. According to her medical history, she had two previous cesarean deliveries and no any additional medical problems including diabetes, thyroid or cardiological disorders. The ethnicity of the patient was Caucasian. The family of the patient did not have any record of cardiovascular abnormalities. The patient did not have a history of smoking or alcohol abuse. In her clinical examination BMI was $35 \mathrm{~kg} / \mathrm{m}^{2}$ before delivery and no any pathology about cardiological system was detected. The patient was also examined by the anesthesiologist and preoperative evaluation was normal by means of cardiological system, systolic and diastolic blood pressure levels. She was delivered via emergent cesarean section with spinal anesthesia and given 2 gr Cefazolin for infection prophylaxy. The newborns were admitted to Neonatal Intensive Care Unit due to prematurity. The patient was given thromboprophylaxy with $40 \mathrm{mg} / \mathrm{d}$ Low Molecular Weight Heparin (LMWH) during hospitalization due to indications of twin pregnancy, emergency cesarean section and high BMI according to Turkish Ministry of Health Labor Management Guidelines. There was no biological evidence to indicate whether this patient had bacterial or viral infection during treatment. She was hospitalized for six days in our inpatient clinics since she complained for post spinal headache. Neurology consultation and cranial tomography were performed and no pathology was detected. She was discharged from the hospital without any complaints with stable hemodynamic condition on the $6^{\text {th }}$ postpartum day. On the $8^{\text {th }}$ postpartum day she was found lying unconsciously on the floor after breast feeding and admitted to emergency department without any cardiac and respiratory functions and the patient was regarded as exitus. For the exact diagnosis, autopsy was performed by Muğla Forensic Medicine Institution. In the autopsy report, morphological examination was consistent with 
postpartum findings. Histopathological examination reported pulmonary congestion, intra-alveolar edema, emphysematous changes, cardiac congestion, perivascular and interstitial edema and focal myocarditis. In conclusion of the autopsy report, the probable cause of death was regarded as 'focal myocarditis' according to histopathological findings.

\section{Discussion}

Maternal death is caused by direct (hemorrhage, hypertension, infections, thrombo emboli) or indirect (cardiac disorders, neoplasms, cerebrovascular disorders) reasons. The crucial point is the predictability and preventability of such reasons. According to reports of the Centers for Disease Control and Prevention, nearly $50 \%$ of maternal deaths were preventable [5]. In our country, Turkey, Maternal Mortality Rate (MMR) decreased from 68 to 23 per 100,000 live births between 1990 and 2008 [6]. It is reported that while sepsis is now the most common cause of direct maternal death, cardiac disease was already the leading cause of indirect maternal death in United Kingdom in 2006 - 2008 [4]. Herein, we report a sudden death of a woman at eight postpartum days and discuss the probable etiologies and differential diagnoses by reviewing the related literature.

Myocarditis is inflammation of the cardiac muscle and is caused by viruses, bacteria, parasites or fungi, and by toxic materials, or by an unknown origin. Myocarditis has a wide spectrum of clinical courses from full recovery to serious complications including permanent cardiac damage or sudden death by fatal arrhythmic events [7]. Myocarditis is a major cause of sudden unexpected death in infants and young adults [8].

Sudden Adult/Arrhythmic Death Syndrome (SADS) is the death of an adult in which no any cause can be identified and it is reported to be the most common cause of cardiac maternal deaths [9]. SADS is also associated with obesity, cardiac hypertrophy, atherosclerosis and high circulating non-esterified fatty acid concentrations [10]. The present case was asymptomatic by means of a cardiological disorder and the only identified risk factor for SADS was a BMI of $35 \mathrm{~kg} / \mathrm{m}^{2}$.

Peripartum Cardiomyopathy (PPCM) is cardiac failure in the last month of pregnancy or within five months after delivery in the absence of underlying heart disease [11]. It is very rare and occurs in one of 3000-4000 pregnancies. PPCM associated risk factors were advanced maternal age, obesity, chronic hypertension, multiparity, multiple gestation, prolonged to colysis with beta-adrenergic agonists and preeclampsia [12]. The patient had multiple risk factors for PPCM including twin pregnancy, multiparity, and obesity. The underlying mechanisms of PPCM were poorly understood. But myocarditis in PPCM is well described as a mechanism for heart failure and has been demonstrated on endomyocardial biopsy studies [13]. In animal models of PPCM bromocriptine which is a prolactin release inhibitor, was found to have hopeful results in both prevention and treatment of this catastrophic disorder [14]. In the present case maternal mortality occurred in the $8^{\text {th }}$ postpartum day and in breastfeeding period during when prolactin levels are very high. Most women present in the first month postpartum with typical heart failure symptoms such as dyspnea, lower extremity edema, and fatigue. But the patient had no specific symptoms related with heart failure. Also, histopathological autopsy examination reported pulmonary congestion, intra alveolar edema, cardiac congestion, perivascular, interstitial edema and focal myocarditis. These findings also strengthen our suspicion for PPCM. But in the macroscopic report, there were no any findings that can support the presence of dilated Cardiomyopathy.

In conclusion, the present case was asymptomatic by means of a cardiological disorder and the only risk factors were multiple pregnancies, multiparity and a BMI of $35 \mathrm{~kg} / \mathrm{m}^{2}$. The probable cause of death was regarded as 'focal myocarditis' according to histopathological findings of autopsy and myocarditis which is documented in the autopsy report of the present case may actually be a complication in this patient rather than the cause of death.

\section{References}

1. Clark SL, Belfort MA, Dildy GA, Herbst MA, Meyers JA, et al. (2008) Maternal death in the 21st century: causes, prevention, and relationship to cesarean delivery. Am J Obstet Gynecol 199: 36.

2. Zöllner J, Curry R, Johnson M (2013) The contribution of heart disease to maternal mortality. Curr Opin Obstet Gynecol 25: 91-97.

3. Arnoni RT, Arnoni AS, Bonini RC, de Almeida AF, Neto CA, et al. (2003) Risk factors associated with cardiac surgery during pregnancy. Ann Thorac Surg 76: $1605-1608$.

4. Centre for Maternal And Child Enquiries (CMACE) (2011) Saving Mothers' Lives: Reviewing maternal deaths to make motherhood safer: 2006-08. The Eighth Report on Confidential Enquiries into Maternal Deaths in the United Kingdom. BJOG 118: 1-203.

5. Berg CJ, Harper MA, Atkinson SM, Bell EA, Brown HL, et al. (2005) Preventability of pregnancy-related deaths: results of a state-wide review. Obstet Gynecol 106: 1228-1234.

6. World Health Organization (1985) Maternal mortality rates, unpublished report. WHO, Geneva, USA.

7. Eriksson U, Penninger JM (2005) Autoimmune heart failure: new understandings of pathogenesis. Int J Biochem Cell Biol 37: 27-32.

8. Maron BJ, Doerer JJ, Haas TS, Tierney DM, Mueller FO (2009) Sudden deaths in young competitive athletes: analysis of 1866 deaths in the United States, 1980-2006. Circulation 119: 1085-1092.

9. James AH, Jamison MG, Biswas MS, Brancazio LR, Swamy GK, et al. (2006) Acute myocardial infarction in pregnancy: a United States population-based study. Circulation 113: 1564-1571.

10. Jouven X, Charles MA, Desnos M, Ducimetière $P$ (2001) Circulating nonesterified fatty acid level as a predictive risk factor for sudden death in the population. Circulation 104: 756-761.

11. Hilfiker-Kleiner D, Sliwa K, Drexler H (2008) Peripartum cardiomyopathy: recent insights in its pathophysiology. Trends Cardiovasc Med 18: 173-179.

12. Pyatt J, Dubey G (2011) Peripartum cardiomyopathy: current understanding comprehensive management review and new developments. Postgrad Med J 87: 34-39.

13. Ntusi N, Mayois B (2009) Aetiology and risk factors of peripartum cardiomyopathy: a systematic review. Int J Cardiol 131: 168-179.

14. Hilfiker-Kleiner D, Struman I, Hoch M, Podewski E, Sliwa K (2012) 16-kDa prolactin and bromocriptine in postpartum cardiomyopathy. Curr Heart Fail Rep 9: 174-182. 